\title{
A Comparative Study of Conventional versus Digital Impression Taking in Implant Dentistry- A Systematic Review
}

\author{
H. Hussien ${ }^{1}$, N. Mallesh² \\ ${ }^{1}$ Department of Dental, My Dentist, Manchester, UK. \\ ${ }^{2}$ Department of Oral and Maxillofacial Surgery, KIMSU, Bangalore, Karnataka, India.
}

\section{ABSTRACT}

\section{BACKGROUND}

Dental implants are one of the most sought-after choice of treatment for restoration and rehabilitation of missing teeth. However, the accuracy of removable or fixed superstructures over the dental implants is influenced for a major proportion by the impression techniques and materials. This paper discusses the comparison of the accuracy of different conventional impression materials and techniques and the intra oral scanners, used in the second stage of the dental implant treatment. The objective of the paper is to compare the accuracy of impressions obtained using conventional impression and intra oral scanners, in the second stage of the dental implant treatment.

\section{METHODS}

In order to perform the study, a PICO framework was formulated, and the topic of the paper was rephrased accordingly. The PICO framework for the topic of interest chosen is outlined. The keywords were thoroughly searched in scientific databases including the Cochrane Library of Databases, PubMed Central, Wiley Online Library and Google Scholar. The key terms used for the search were dental implants, impression materials, impression techniques, digital impression, intra oral scanners, accuracy and success.

\section{RESULTS}

Among the different impression materials taken into the comparative study for the conventional impressions, it was observed that polyvinyl siloxane exhibited a dimensional accuracy slightly greater than polyether. However, light body putty exhibited greater accuracy than medium body putty. Various impression techniques were compared to evaluate the dimensional accuracy of the impression. It was observed that the open tray technique and splinted impression technique produced a more dimensionally stable impression than the other techniques. Among conventional and digital impressions, it was observed that intra oral scanners exhibited a greater degree of accuracy.

\section{CONCLUSIONS}

This study is to comparatively assess the accuracy of conventional and digital impression of dental implants in the second stage of treatment. Although accuracy is greater in digital impressions since there is no likelihood of distortions or dimensional changes or any delay in pouring of models; however, incomplete capture of sub-gingival details, image obscured due to bleeding are possible with intra-oral scanners. Hence, between conventional and digital impressions, the dentist may gradually need to upgrade to the technology and advancements.

\section{KEY WORDS}

Dental Implants, Impression Materials, Impression Techniques, Digital Impression, Intra Oral Scanners, Accuracy, Success
Corresponding Author:

Dr. Natashekara Mallesh, \#48, Sarathy Apt., Flat \#001A, $13^{\text {th }}$ Cross, Malleshwaram, Bangalore-560003, Karnataka, India. E-mail: drnshekhar@yahoo.co.in

DOI: $10.14260 /$ jemds/2019/729

Financial or Other Competing Interests: None.

How to Cite This Article:

Hussien H, Mallesh N. A comparative study of conventional versus digital impression taking in implant dentistry- a systematic review. J. Evolution Med. Dent. Sci. 2019;8(44):3362-3367, $10.14260 / \mathrm{jemds} / 2019 / 729$

Submission 27-07-2019, Peer Review 20-10-2019, Acceptance 26-10-2019, Published 04-11-2019.

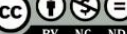




\section{BACKGROUND}

Dental implants are a novel, evolving and convenient method for replacement of missing teeth, the success of which is determined by many factors that are not limited to those factors under the control of the surgeon performing the treatment. While patient selection, selection of implant type, size and form, recommending appropriate superstructures as necessary and pre-surgical treatment planning play an important role, these decisions call for sound knowledge and clear evidence to back up the clinical decision, without which the wrong choice in any one of the treatment steps may prove implant failure. However, one important step in the dental implant treatment that has an influential role in the outcome of the treatment is the impression. The dimensional accuracy and outcome of the impression are determined by various variables, such as the choice of impression material, choice of impression technique- conventional versus digital, the presence of coping, open or closed tray technique and angulation of implants.

\section{METHODS}

\section{Methodology and Study Design}

In order to perform the study, a PICO framework was formulated, and the topic of the paper was rephrased accordingly. The PICO framework for the topic of interest chosen is outlined.

\section{PICO Framework}

P- Implant impressions.

I - Comparison of accuracy.

C- Literature review of scientific databases.

0- Study to facilitate knowledge sharing and avoid/minimise the errors in impression procedure.

\section{Databases Searched}

The keywords were thoroughly searched in scientific databases including the Cochrane library of databases, PubMed Central, Wiley online library and Google Scholar. The key terms used for the search were dental implants, impression materials, impression techniques, digital impression, intra oral scanners, accuracy and success.

\section{Eligibility Criteria}

The inclusion criteria for the study were observational studies pertaining to the accuracy of implant impression materials and techniques, conventional impression techniques, digital impressions, impressions taken with implant fixture, studies conducted in the mandibular arch for partial edentulousness, and the age group of 30 to 50 years. Exclusion criteria formulated were complete edentulous, dual arch impressions and the All-on-four technique.

\section{Search Strategy}

Thorough screenings of the literatures obtained were performed manually and electronically, omitting the duplicates were performed for a total of 258 literatures. During the screening step, irrelevant scientific articles or those that did not fit into the inclusion criteria were removed.

\section{Data Extraction}

Only the articles that fulfilled the chosen criteria were considered, and a total of 22 literatures were found to be relevant to the topic of interest and were chosen as the reference list for writing the study for the topic chosen.

\section{RESULTS}

From the above detailed reviewing of various pertinent scientific articles obtained from reliable scientific databases, it is evident that the dimensional accuracy and outcome of the impressions taken for dental implant treatment is influenced by variable measures such as the impression method (Conventional or digital), impression material, impression techniques (e.g. open tray or closed tray), transfer or pick up impressions, splinted or non-splinted techniques, relation and angulation of adjacent implants, and abutments with or without impression coping.

Among the 4 studies found pertaining to digital impressions or intra oral scanners, it was observed that they reproduce a nearly accurate and true replica of the intra oral teeth and structures. They are more accurate because there is no time loss between the capture of data and reproduction of data and there is no transfer delay to the lab or damage due to handling. Since it is more convenient to the patient and reduced chair side time for the doctor, intra oral scanners are likely to grow into the market in future. Deviations are observed in maxillary and mandibular $1^{\text {st }}$ molar region when different scanners were compared. One study also concluded that they also tend to produce a greater trueness and precision than conventional impressions.

Among the 5 studies found pertinent to the impression materials and their impact on accuracy, it has been observed that the choice of impression material has no significant impact on the accuracy of the impressions. Two studies have favoured polyvinyl siloxane, and 1 study found polyether produced more accurate impressions. However, amongst the 2 impression materials considered for the discussion (Polyvinyl siloxane and polyether), a slightly greater dimensional accuracy was observed in putty light body polyvinyl siloxane impressions than in medium body polyether. However, the difference is not significant to conclusively prove the point of the supremacy of one material over the other.

Among the impression techniques, while 3 studies have found closed tray technique to produce more accurate impressions than the open tray technique, a conflicting result was observed in 2 studies favouring the open tray technique, while 1 study has indicated that the impression technique has no influence on the accuracy of implant impressions, thereby giving rise to the dilemma of the choice of open versus closed tray techniques. Between the choice of impressions using splinted or non-splinted techniques, 4 studies have proved that the splinted technique produces impressions that are more dimensionally accurate than those obtained by the nonsplinted technique. Among the choice of transfer versus pick up impressions to transfer the copings of dental implants, it has been observed that only 2 relevant studies were sourced, and they did not conclusively prove the accuracy of one impression method over the other. 


\section{DISCUSSION}

The main objective of this study is to provide a broad perspective on the comparative study of the dimensional stability, surface details and accuracy of conventional and digitally recorded impressions in dental implant treatment, to enable the dentist to perform every step of the dental implant treatment with negligible errors and as accurate as possible. For the purpose of knowledge sharing, study of relevant literatures was performed to arrive at a competing and conclusive result.

\section{Conventional Impression/Impression Materials}

Among the many impression materials used such as the impression plaster, alginate, wash impression (zinc oxide eugenol), polysulphide, condensation silicone and other materials, polyvinyl siloxane and polyether materials are used often. So, studies comparing the dimensional stability of these two materials (polyvinyl siloxane and polyether) will be elaborated. Studies comparing these materials showed no significant difference in the accuracy of the impressions obtained.(1,2)

\section{Polyether versus Vinyl Polysiloxane}

Lee $\mathrm{H}$ et al. conducted a study on the effect of subgingival depth of implant placement and the dimensional accuracy of different consistencies of polyvinyl siloxane and polyether materials. It was concluded that putty light body combined with polyvinyl siloxane impressions were more dimensionally stable than medium body polyether.

Another study was conducted to study the influence of implant position, tray type and impression material on the outcome of the accuracy of implant impressions by Gokcen Rohlig B et al. in 2014. It was observed that between the polyvinyl siloxane medium body and polyether impression materials, there was no discrepancy between the materials and in the accuracy of the impression obtained (3)

\section{Impression Techniques Studied}

While the common dental implant impression materials have been reviewed in detail, a significant portion of the accuracy is determined by the impression technique. The most commonly used impression techniques are splinted or nonsplinted methods, open tray or closed tray techniques, transfer or pick- up impressions and other recent advances. However, extensive studies have already summarised the accuracy and comparison of each of the dental implant impression techniques.

\section{Splint Versus Non-Splint Technique}

The impressions for the dental implant with copings, require an accuracy of the highest order. When the number of superstructures involved are increased, a greater degree of accuracy is required to obtain an impression which enables cast fabrication with negligible errors or distortions. $(4,5,6)$ Heeje Lee et al conducted a systematic review to study the accuracy of implant impression techniques and the clinical factors affecting the accuracy of the impression. It was observed that although no study definitively pointed out the superiority of either of the techniques, the splinted technique displayed greater accuracy than non-splinted methods in the study, with seven studies supporting the splinted method as compared to the three studies supporting the non-splinted technique (7). However, splinted technique exhibits a greater accuracy in dental implants treatment transfer of impression copings.

\section{Open Tray versus Closed Tray Technique}

When multiple implant impression copings or a full arch rehabilitation is performed, the open tray technique of impression or closed tray technique is the most commonly used form of impression technique. Balouch $\mathrm{F}$ et al. conducted an experimental study to determine the dimensional accuracy of the open and closed tray implant impression technique in angled implants. It was observed that the measurement of dimensional accuracy and coefficient of variation displayed a significant difference in the assessment, thereby concluding that the closed tray technique produces a more dimensionally accurate impression with a lower coefficient of variation than the open tray technique. $(5,8,9,2)$

Yet another in vivo comparative research study was conducted by Gallucci GO et al. in 2011 to compare the accuracy outcomes of the open tray and closed tray impression techniques in partially edentulous patients treated with implants. The study measured the outcomes subjectively and objectively. While subjective measures of patient comfort showed no difference in the outcome, objective measurement of the 2 techniques was made using micro-computed tomography scanning. The quantitative measures showed no significant difference in the open and closed tray techniques when the abutments of the implants were placed parallel or when the angulation was less than 10 degrees.(10)

\section{Transfer versus Pick Up}

The difference between these impression techniques is that the transfer technique is performed with a closed tray, and the impression copings are left intraorally, whilst in the pickup impression technique, the open tray method is used and the copings are unscrewed to be removed along with the impression. $(4,5,11)$ However, when comparing the accuracy between the two techniques, only two relevant studies were found, and they were not conclusive in proving the accuracy of the transfer impression technique.

\section{Angulation}

To study the influence of the angulation of implants on the accuracy of the impression, Reddy $S$ et al. conducted a study to compare the accuracy of impressions taken with polyvinyl siloxane and polyether in parallel and angulated implants. It was observed that the impression material had no influence on the accuracy of parallel implants while a similar result was concluded in implants with angulations of 10- 15 degrees (12). However, implants with angulations greater than 15 degrees were considered for this study, thereby not conclusively proving the point. In order to further study the influence of angulation of implants on the accuracy of impressions, Vojdani $\mathrm{M}$ et al. conducted a study comparing 3 impression materials (Polyvinyl siloxane, polyether and vinyl siloxane ether), on parallel and non-parallel implants with angulations up to 30 degrees. It was observed that the choice of impression materials among the 3 mentioned did not affect the dimensional accuracy of impressions in cases of parallel 
implants.(13) However, in non-parallel implants, polyvinyl siloxane exhibited greater dimensional accuracy of impressions than vinyl siloxane ether and polyether.

Parameswari G. et al. conducted an extensive in vitro study to evaluate the accuracy of various impression materials and impression techniques in recording impressions for multiple implants placed unilaterally in a partially edentulous mandible. The study established its conclusion that the impression materials, polyvinyl siloxane and polyether, produced impressions of similar dimensional accuracy. Whilst impression techniques, both open and closed tray, had no influence of parallel implants. For nonparallel implants of angulation up to 15 degrees, the open tray custom impression technique produced a more dimensionally accurate impression.(14) The study thereby establishes that angulation of implants has an influence on the accuracy of the impressions.

\section{Coping}

To understand the influence of coping on the accuracy of the impression details, Kwon JH et al. conducted a study to compare the dimensional accuracy of casts fabricated from impressions taken both with and without copings. The group was split into a coping, and no coping group, and the impressions were made. The linear and rotational distortions of each implant site in the outcome were measured and calculated with the Mann Whitney U test.(6) It was observed that greater distortion values were recorded in impressions with no coping, than within impressions with coping, thereby indicating the influence of coping on the dimensional accuracy of implant impressions. However, a single study with linear measurements does not necessarily prove the significance of impressions with dental implant coping. One other study was conducted by Rashidan N et al. n 2012, to study the influence of implant coping shapes on the accuracy of the impression. The study concluded that the shape of the impression coping had more influence on the impression inaccuracy than the impression technique or material.(15) However, the study was not conducted with various impression techniques to produce a comparative conclusion of the coping shape and impression technique.

\section{Other Techniques}

More recent advancements of impression techniques is the snap-fit plastic impression coping technique wherein the closed tray technique is used, but the copings are removed with the impression. (7) Further studies are needed to evaluate the efficacy and accuracy of this implant impression technique.

To compare the efficacy and accuracy of impressions obtained by various impression methods and techniques, Papaspyridakos et al. conducted a comparative review study in vitro and in vivo, with impressions obtained by open and closed tray techniques, splinted and non-splinted techniques, as well as polyvinyl siloxane and polyether impression materials. It was observed that the dimensional accuracy of the dental implant impressions was greater in splinted techniques than in non-splinted techniques. Furthermore, the impressions obtained by open tray techniques were more accurate than the closed tray techniques. The study also concluded that the impression material had no influence on the accuracy of the impression for dental implants (16). The study was extensive and covers important factors influencing the outcome and dimensional stability of impressions taken for dental implant treatment. However, the study does not take into account the influence of coping, angulations of implant abutments, the inter-implant distance on the accuracy of impressions taken for dental implant treatment, and hence cannot conclusively establish the factors influencing the outcome of implant impressions. Also, the variables taken for this comparative study are 2 comparable measures, thereby questioning the credibility of the study, since third or fourth measures or methods are available or evolving. This may prove to be more significant than the measures used for comparison.

One other study was conducted by Sonam Gupta et al., to comparatively evaluate the different impression techniques and impression materials on the accuracy of open tray implant impressions. It was observed that polyether produced greater dimensionally accurate impressions than the polyvinyl siloxane (17). Also, among the impression trays, they seemed to have produced no statistically significant differences when custom and stock trays were used, thereby indicating that impression trays had no influence on the outcome of the accuracy of impressions.

\section{Digital Impressions/Intra Oral Scanners}

Digital impressions are the recent advancements in recording of impressions. They are recorded by means of intra oral scanners that utilise the fibre optic light source and a sensor to capture the details and reproduce it immediately without a delay on screen. This can then be sent to the lab for fabrication of prosthesis, or used for assessment of the arches/occlusion and ridges. These scanners may produce a monochromatic image or multichromatic image depending on the chroma range in the scanner. Multichromatic scanners also provide the option of automatic shade selection for the prosthesis, recording of bite and the associated structures as required. Various studies have been performed to study the accuracy of these intra oral scanners.

A literature review of intra oral scanners in dentistry wad performed by Franceso Mangano et al in 2017. The literature review concluded that digital impressions have reduced the working time needed to transfer the patient details to the lab, improved the communication with laboratory technician, reduced discomfort for the patient, reduced armamentarium and more predictable treatment outcome (18). Although intra oral scanners offer more advantages than the conventional impressions, there are possibilities of inability to capture sub gingival details or inaccurate capture of data due to bleeding and they are comparatively more expensive than other impression techniques. Further upgradation may be necessary to rule out these drawbacks of intra oral scanners.

Another study that compared the accuracy of different intra oral scanners using simulators was conducted by Hye Nan- Park et al in 2017. The scanners used were Identica Blue, 3Shape TRIOS and Carestream C3500. Each of the simulator was scanned 10 times with each scanner. Specific points referring to the corresponding teeth in the model were labelled, such as D3 for Canine, D6 for 1st molar, and D7 for $2^{\text {nd }}$ molar. The distance between these points on either side of the model were compared statistically using Kruskal Wallis test. 


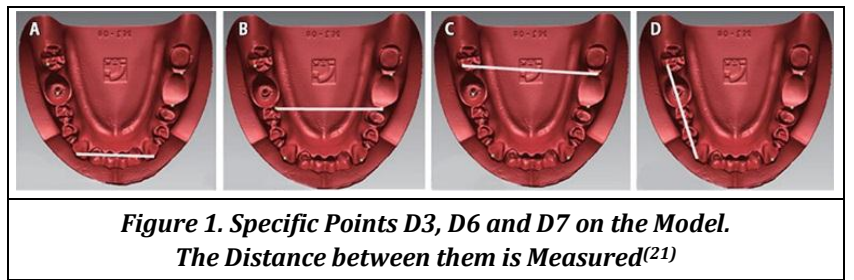

It was observed that no difference was found in the intra oral simulators. However, the scanners showed deviations at D6 AND the accuracy was highest in TRIOS, followed by Identica Blue and deviations were more in Carestream (19). Utilising intra oral simulators have made the study highly reliable and further studies with different objectives may produce more scientifically relevant results. However, the deviations at D6 requires to be upheld by the Intra oral scanner developers.

To study the accuracy of different intra oral scanners, Ji Hye Chun et al conducted a study in 2017 that evaluated the interdental space between the mandibular incisors. Scanners used in this study were Sensable S3, iTero1, iTero Orthodontic version, TRIOS2 and 3Shape TRIOS. 4 models with spacing of $0,1,3$ and $5 \mathrm{~mm}$ spacing were chosen for the study. Data was captured with various intra oral scanners and computed for statistical analysis. Data obtained using Sensable S3 was considered the standard and rest of the data were compared with Sensable S3. It was observed that TRIOS produced more accurate results than iTero and deviations were more in Mandibular $1^{\text {st }}$ molar region. However, both iTero and TRIOS produced scientifically admissible results in relation to mandibular anterior region (20). The study was primarily conducted for orthodontic scanning and diagnostic purpose and the evidence of deviation in mandibular molar region needs to quantified with meta-analysis so that there are no errors while fabricating prosthesis in molar region.

To study the precision and trueness of Intra Oral Scanners (IOS), Francesco Mangano et al conducted a study on single and multiple implant impressions. The scanners used in the study are Carestream CS3600, 3shape TRIOS, CEREC Omnicam, DWIO Dental Wings and Planmeca Emerald. Scenario taken into consideration were partially edentulous maxilla to be restored with Single Crowns (SC) and Partial Prosthesis (PP) and completely edentulous maxilla to be restores with Full Arch Prosthesis (FA). Plaster models of these scenarios were obtained and were scanned 10 times using each of the 5 IOS. The results were statistically analysed. It was concluded that CS3600 displayed the highest trueness and precision among the 5 scanners followed by TRIOS, DWIO, Omnicam and Emerald sequentially.(21) Although differences in the accuracy between different scanners were present, they were not taken into account in this study.

\section{Recommendations}

The extensive study of the literature and the results with regards to the topic obtained by scientific data and research have been established. While not all results have been conclusively evident, they have sufficiently established results that are backed up, however further research is required. Among the 5 studies that elaborated on the choice of impression material, no significant difference was observed between polyvinyl siloxane and polyether. Hence, they may require more extensive studies conducted in vivo or on impression materials with a change in their composition, to prove the point of dimensional accuracy. Also, the results of open versus closed tray techniques have been controversial, necessitating the need for more studies in this regard. The choice of splinted versus non-splinted impression techniques has been conclusively established, favouring the splinted technique for a greater dimensionally accurate impression. However, among the decision of transfer versus pick up impression methods, it has been observed that no studies have conclusively established the choice of transfer or pick up impression method, and hence requires further extensive and elaborate studies to arrive at a conclusion.

Among the conventional and digital impressions, it is observed that intra oral scanners are a great advantage in produced accurate, true and precise replica of intra oral details. Also, they are more patient compliant, especially for those with gag reflex, and reduced mouth opening. Also, the choice of intra oral scanner plays a major role since the degree of accuracy and percentage of deviations varies with each scanner. Although the digital impressions are fraught with few cons, overcoming them will make them the implant dentistry treatment and planning more easy, predictable, helps in accurate planning and execution.

\section{CONCLUSIONS}

Dental implants have been in use for nearly 3 decades and have become the bread and butter of prosthetic rehabilitation. Hence, dental practitioners require extensive research studies to make an informed decision and to update themselves with the recent advancements continuously. Digital impressions provide an advantage over the conventional impressions. Among the intra oral scanners, Carestream and TRIOS exhibit a greater degree of accuracy and precision. Among the conventional impression materials, polyvinyl siloxane exhibits a slightly higher dimensional accuracy of the obtained impression, when compared to polyether. Among the impression techniques, the open tray technique and splinted technique impression method produce a more dimensionally accurate impression, provided the implants are parallel or with lesser axial angulation (less than 15 degrees), and appropriate coping shapes are selected.

\section{REFERENCES}

[1] Enkling N, Bayer S, Jöhren P, et al. Vinylsiloxanether: a new impression material. Clinical study of implant impressions with vinylsiloxanether versus polyether materials. Journal of Clinical Implant Dentistry and Related Research 2012;14(1):144-51.

[2] Lee H, Ercoli C, Funkenbusch PD, et al. Effect of subgingival depth of implant placement on the dimensional accuracy of the implant impression: an in vitro study. J Prosthet Dent 2008;99(2):107-13.

[3] Gökçen-Rohlig B, Ongül D, Sancakli E, et al. Comparative evaluation of the effects of implant position, impression material, and tray type on implant impression accuracy. Implant Dent 2014;23(3):283-8. 
[4] Filho HG, Mazaro JV, Vedovatto E, et al. The accuracy of impression techniques for implants. Part 2 - Comparison of splinting techniques. Journal of Prosthodontics 2009;18(2):172-6.

[5] Wöstmann B, Rehmann P, Balkenhol M. Influence of impression technique and material on the accuracy of multiple implant impressions. Int J Prosthodont 2008;21(4):299-301.

[6] Jahandideh Y, Moghaddam AA, Pourkhalili H. Accuracy of implant abutment level impression with and without impression coping. J Contemp Dent Pract 2018;19(11):1352-7.

[7] Lee H, So JS, Hochstedler JL, et al. The accuracy of implant impressions: a systematic review. Louisiana State University, School of Dentistry, New Orleans, La; University of Rochester Eastman Dental Center, Rochester, NY. J Prosthet Dent 2008;100:285-91.

[8] Balouch FA, Jalalian EA, Nikkheslat M, et al. Comparison of dimensional accuracy between open-tray and closedtray implant impression technique in $15^{\circ}$ angled implants. J Dent Shiraz Univ Med Sci 2013;14(3):96-102.

[9] Siadat H, Saeidi Z, Alikhasi $M$, et al. Comparative evaluation of the effect of impression materials and trays on the accuracy of angulated implants impressions. J Clin Exp Dent 2018;10(11):e1096-e102.

[10] Gallucci GO, Papaspyridakos P, Ashy LM, et al. Clinical accuracy outcomes of closed-tray and open-tray implant impression techniques for partially edentulous patients. Int J Prosthodont 2011;24(5):469-72.

[11] Wenz HJ, Hertrampf K. Accuracy of impressions and casts using different implant impression techniques in a multi-implant system with an internal hex connection. Int J Oral Maxillofac Implants 2008;23(1):39-47.

[12] Reddy S, Prasad K, Vakil H, et al. Accuracy of impressions with different impression materials in angulated implants. Niger J Clin Pract 2013;16(3):279-84.
[13] Vojdani M, Torabi K, Ansarifard E. Accuracy of different impression materials in parallel and nonparallel implants. Dent Res J (Isfahan) 2015;12(4):315-22.

[14] Parameshwari G, Chittaranjan B, Sudhir N, et al. Evaluation of the accuracy of various impression techniques and impression materials in recording multiple implants placed unilaterally in a partially edentulous mandible - an in vitro study. J Clin Exp Dent 2018;10(4):e388-e95.

[15] Rashidan N, Alikhasi M, Samadizadeh S, et al. The accuracy of implant impressions with the different impression coping types and shapes. Clin Implant Dent Relat Res 2012;14(2):218-25.

[16] Papaspyridakos P, Chen CJ, Gallucci GO, et al. Accuracy of implant impressions for partially and completely edentulous patients: a systematic review. Int J Oral Maxillofac Implants 2014;29(4):836-45.

[17] Gupta S, Narayan AI, Balakrishnan D. In vitro comparative evaluation of different types of impression trays and impression materials on the accuracy of open tray implant impressions: a pilot study. Article ID 6306530, Int J Dent 2017;(2017):1-8.

[18] Mangano F, Gandolfi A, Luongo G, et al. Intraoral scanners in dentistry: a current literature review. BMC Oral Health 2017;17(1):149.

[19] Park HN, Lim YJ, Yi WJ, et al. A comparison of the accuracy of intraoral scanners using an intraoral environment simulator. J Adv Prosthodont 2018;10(1):58-64.

[20] Chun JY, Tahk JH, Chun YS, et al. Analysis on the accuracy of intraoral scanners: the effects of mandibular anterior interdental space. Appl Sci 2017;7(7):719. www.mdpi.com/journal/applsci

[21] Mangano FG, Hauschild U, Veronesi G, et al. Trueness and precision of 5 intraoral scanners in the impressions of single and multiple implants: a comparative in vitro study. BMC Oral Health 2019;19(1):101. 